\title{
Image Segmentation using Clustering Algorithms
}

\author{
P. Srinivasa Rao \\ Professor \\ Andhra University College of \\ Engineering, Visakhapatnam.
}

\author{
K. Suresh \\ Assistant Professor \\ Anil Neerukonda Institute of \\ Science \& Technology
}

\author{
B. Ravi Kiran \\ Sr. Assistant Professor \\ Anil Neerukonda Institute of \\ Science \& Technology.
}

\begin{abstract}
Pictures are considered as a standout amongst the most imperative medium of passing on information. Understanding pictures and separating the data from them such that the data can be utilized for different undertakings is a critical part of Machine learning. Picture division is the methodology of separating the given picture into districts in light of a few properties. The grouping alludes to methodology of collection tests so that examples are comparative inside every gathering These gatherings are called groups. This undertaking addresses the issue of portioning a picture into distinctive districts. We investigate this issue by utilizing calculations like K-means and chart based calculation standardized cuts. We are going to look at these calculations by their time complexities.
\end{abstract}

\section{Keywords}

Image processing, Image Segmentation, K-Means algorithm, Normalized Cut algorithm

\section{INTRODUCTION}

Images are considered as a standout amongst the most critical medium of passing on information.Understanding pictures and disparating the data from them such that the data can be utilized for different errands is a vital part of Machine learning. A sampleswould be the utilization of pictures for route of robots. Different applications like separating censure tissues from body examines and so on structure indispensable piece of Medical judgment. One of the preliminary phases in heading of comprehension pictures is to section them and discover diverse questions in them. To do this, pinnacles like the histogram plots and the recurrence area change can be utilized[4].

Image segmentation is, the splitting of an image into regions or groups, which dovetail with different objects or parts of objects. Each pixel in an image is alloted to one of a number of these groups. A better segmentation is conventional one in which pixels in the same category have nearby grey scale of multivariate values and form a connected region, neighboring pixels which are in different categories have different values[3].

The process of organizing the objects into groups based on its attributes is called clustering. A collection of objects which are "similar" between them and are "dissimilar" to the objects which belongs to other clusters is called clustering. An image can be categorized which based up on keyword (metadata) or its description. To make the segmentation more efficacious a variety of clustering techniques have been introduced.

Remaining sections of the paper describes as follows, section 2 describes the related works based on various subsections like existing works related to image segmentation algorithms. Section 3 compares the both algorithms, in 4 results and observations and finally concludes the paper in section 5 .

\section{IMAGE SEGMENTATION ALGORITHMS}

The procedure of dividing a digital image into multiple segments is known as image segmentation. The main aim of segmentation is to clarify and/or change the characterization of an image into something that is more meaningful and simple to survey. Image segmentation is typically used to detect objects and boundaries (lines, curves, etc.) in images[4]. Here we can cluster the image by image segmentation methods. They can be detected by using intensity values of clusters. In this research we are using two algorithms on an image in image segmentation. They are KMeans algorithm from clustering method and N-Cut algorithm from graph partitioning method.

\subsection{K-Means algorithm[1][4]:}

In image segmentation K-Means algorithm is from clustering methods. K-Means calculation is an unsupervised grouping estimating that orders the data information focuses into various classes in view of their inborn separation from one another. The computation expect that the information highlights structure a vector space and tries to discover regular grouping in them. The focuses are grouped around centroids $\mathrm{i}=1: \mathrm{k}$ which are gotten by minimizing the goal where there are $\mathrm{k}$ groups $\mathrm{S}_{\mathrm{i}}, \mathrm{i}=1 ; 2 ;: ; \mathrm{k}$ and $\mathrm{i}$ is the centroid or mean purpose of every last one of focuses $x_{j}$ to $S_{i}$.

As a piece of this venture, an iterative adaptation of the calculation was actualized. The calculation takes a 2dimensional picture as info. Different ventures in the calculation are as per the following:

1. Figure the force distribution(also called the

histogram) of the intensities.

2. Instate the centroids with $\mathrm{k}$ irregular intensities.

3. Rehash the accompanying ventures until the

bunch marks of the picture does not change

any longer.

4. Bunch the focuses taking into account separation

of their intensities from the centroid intensities.

5. Figure the new centroid for each of the clusters.

where $\mathrm{k}$ is a parameter of the computation (the quantity of bunches to be discovered), i repeats over all the intensities, $j$ emphasizes over all the centroids and $\mathrm{i}$ are the centroid intensities. 


\subsection{Normalized Cut algorithm [2]:}

The standardized cut calculation can bunch an arrangement of components built just in light of the estimations of a closeness measure between all conceivable sets of components. The methodology is a phantom bunching strategy. It is in view of the properties of eigenvectors from a network figured utilizing the likenesses between every sets of components.

In the standardized cut plan, the grouping is seen as a diagram apportioning issue. The hubs of the diagram are the components and the weights on the chart edges joining two hubs are the similitudes measured between the two comparing components. We utilize the similitude measure depicted as a part of the earlier area. We try to segment the picture into subgraphs with high likenesses between the hubs of the same sub graphs and a low similitudes between hubs from diverse sub graphs.

Image division can likewise be seen as an ideal parceling of a graph. The picture is exhibited as a weighted undirected chart $\mathrm{G}=(\mathrm{V} ; \mathrm{E})$. This picture can be dispensed into two sub graphs $\mathrm{A}$ and $\mathrm{B}$ by displaying the allotment as minimizing the cut as characterized underneath: where $w(i ; j)$, the weight of every edge is a ability of the likeness between hubs I and $\mathrm{j}$. However the base cut criteria favors cutting little arrangements of disengaged hubs in the graph.To beat these peculiarities we can utilize an adjusted expense capacity, Normalized Cut as characterized below. The federating reverence, $\operatorname{assoc}(\mathrm{A}, \mathrm{V})$, is the agglomerating association from hubs $\mathrm{A}$ to all hubs in the chart. N-cut esteem won't be small for the cut that segments disengaging focus, on the grounds that the cut quality will be a substantial rate of the aggregate association from that set to the others. Given a segment of a diagram $\mathrm{V}$ into two disjoint corresponding sets $\mathrm{A}$ and $\mathrm{B}$, let $\mathrm{x}$ be a $\mathrm{N}=\mathrm{jV}$ jdimensional affirmation vector, $x i$ from hub $i$ to all various hubs. Necessities on y explained from the condition on the relating pointer vector $\mathrm{x}$. The second littlest eigenvector of the sum up framework fulfills the ordinariness imperative. $y 1$ is a genuine esteemed answer for our standardized cut issue.

\section{COMPARISON BETWEEN CLUSTERING AND GRAPH PARTITIONING METHODS}

Picture division result is then conveyed by gathering on the eigenvector. Each of the part in the figured eigenvector contains portrayal worth contrasts with each of the pixel in regards to spatial and sparkle (pixel power) estimation in the photo. Ideally, eigenvector with the tiniest nonzero eigen value is chosen to perform grouping on it. In this paper, eigenvector groups to the eigen value is picked for k-means gathering count to do the grouping on the eigen vector[1]. After the batching process on the eigenvector of a picture, division result is finally obtained considering the assembled eigenvector. This grouped eigenvector is then mapped by bit in the picture. In any case when K-means is executing it built up in light of k quality. It can group just for littler estimations of $\mathrm{k}$ to the calculations which give great results. For greater estimations of $\mathrm{k}$, the division is over whelming, numerous groups develop in the pictures at particular spots. This is on the grounds that Euclidean separation is not a decent metric for division techniques. A percentage of the best calculations like the chart based $\mathrm{N}$-Cuts give better results for an enormous estimations of $\mathrm{k}$. One normal contrast associating the N-cuts and bunching calculations is that grouping calculations consider the pixels with comparatively close force values as fitting in with one fragment, regardless of the fact that they are not situationally close. N-cuts delibrates such zones as diverse fragments. N-Cuts usage is computationally complicate [2]. The Eigen value technique get a handle on a long-lasting for a full scale image. Pictures must be resized to show the indication of our improvement results. The creators are utilized for implementing of $\mathrm{N}$-Cuts to conceive the outcomes.

These results are existed by using the formulas:

In $k$-means clustering aims to split the image as $n$ results into $k(\leq n)$ sets $\mathbf{S}=\left\{S_{1}, S_{2}, \ldots, S_{k}\right\}$ so as to decline the withincluster sum of squares (WCSS). In another way, its objective is to find:

$\underset{\mathrm{S}}{\operatorname{argmin}} \sum_{i=1}^{k} \sum_{X \varepsilon S \mathrm{i}}\left\|X-\mu_{\mathrm{i}}\right\|^{2} \ldots \ldots(1)$

In Normalized cuts[2]:

Let the weighted graph be $G=(V, E, w)$. Let $A$ and $B$ are the two subsets of vertices.

Let:

$w(A, B)=\sum_{i \in A, j \in B} w_{\mathrm{ij}}$
$\operatorname{ncut}(A, B)=\frac{w(A, B)}{w(A, V)}+\frac{w(A, B)}{w(B, V)} \ldots \ldots$.

$\operatorname{nassoc}(A, B)=\frac{w(A, A)}{w(A, V)}+\frac{w(B, B)}{w(B, V)}$
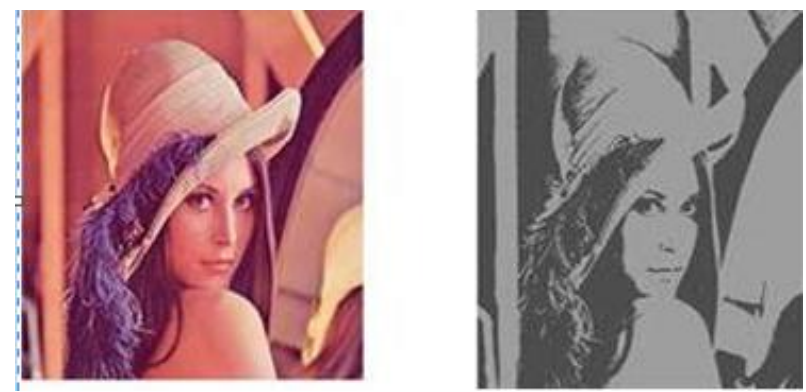

By comparing the results regarding these values we compare the algorithms in image segmentation on an image. By depending on theseformulas which are based on their eigen vectors of an image.

\section{RESULTS AND OBSERVATIONS}

We applied the partitioning algorithm to gray-scaled images. We have implemented k-means and normalized cuts and time complexities are prepared for both alorithms.

$\mathrm{K}$ means : for $\mathrm{k}=2$

N-CUTS: FOR K=2

Fig:FOR K=2, K-MEANS IS COMPUTATIONALLY FASTER THAN N-CUTS.

$\mathrm{T}=4.0871$ SEC FOR K-MEANS

$\mathrm{T}=26.4688$ SEC FOR N-CUTS 


\section{CONCLUSION}

We executed the K-means clustering calculation and utilized it for potentiality division. For small estimations of $\mathrm{k}$ the calculations give great results. For greater estimations of k, the division is tangible, numerous varieties develop in the pictures at distinctive spots. This is an account of Euclidean separation is not a decent metric for division methods. A chunk of the best calculations like the diagram based N-Cuts give better results for an enormous estimations of $\mathrm{k}$. One regular contrast in the middle of $\mathrm{N}$-cuts and bunching calculations is that grouping calculations ponder the pixels with comparatively close force values as fitting in with one fragment, regardless of the possibility that they are not situationally close. N-cuts consider such territories as diverse segments. N-Cuts execution is computationally inter twist. The eigen value technique get a handle on a long-lasting for a full scale picture. Pictures must be resized to show signs of refinement results. We utilized the author's usage of N-Cuts to conceive the outcomes. We executed the K-means clustering calculation and utilized it for potentiality division. For small estimations of $\mathrm{k}$ the calculations give great results. For greater estimations of $\mathrm{k}$, the division is tangible, numerous varieties develop in the pictures at distinctive spots. This is an account of Euclidean separation is not a decent metric for division methods. A chunk of the best calculations like the diagram based $\mathrm{N}$-Cuts give better results for an enormous estimations of $\mathrm{k}$. One regular contrast in the middle of $\mathrm{N}$-cuts and bunching calculations is that grouping calculations ponder the pixels with comparatively close force values as fitting in with one fragment, regardless of the possibility that they are not situationally close. N-cuts considers such territories as diverse segments. N-Cuts execution is computationally intertwist. The eigen value technique get a handle on a long-lasting for a full scale picture. Pictures must be resized to show signs of refinement results. We utilized the authors usage of $\mathrm{N}$-Cuts to conceive the outcomes.

\section{REFERENCES}

[1] Ms. Chinki Chandhok, Mrs.Soni Chaturvedi, Dr. A. A. Khurshid Image Segmentation using K-means Clustering Algorithm International Journal of Information Technology (IJIT), Volume - 1, Issue - 1, August 2012.

[2] Jianbo Shi and Jitendra Malik, Member, IEEE. Normalized Cuts and Image Segmentation IEEE TRANSACTIONS ON PATTERN ANALYSIS AND MACHINE INTELLIGENCE, VOL. 22, NO. 8, AUGUST 2000.

[3] R.Yogamangalam et al. Segmentation Techniques Comparison in Image Processing International Journal of Engineering and Technology (IJET).

[4] Oliver, A.; Munoz, X.; Batlle, J.; Pacheco, L.; Freixenet, J., "Improving Clustering Algorithms for Image Segmentation using Contour and Region Information," Automation, Quality and Testing, Robotics, 2006 IEEE International Conference on, vol.2, no., pp.315,320, 25-28 May 2006.

[5] Mei Yeen Choong; Wei Yeang Kow; Yit Kwong Chin; Angeline, L.; Teo, K.T.K., "Image segmentation via normalised cuts and clustering algorithm," Control System, Computing and Engineering (ICCSCE), 2012 IEEE International Conference. 